\title{
Chinese Herbal Medicine for Treatment of Hiv/aids- associated Diarrhea: a Protocol of Systematic Review and Meta-analysis of Randomized Clinical Trails
}

\section{Bai-lin Chen}

Beijing University of Chinese Medicine

Ming-zhu Zhang

Beijing University of Chinese Medicine

Zi-wei Huang

Beijing University of Chinese Medicine

Hong-rui Zhang

Beijing University of Chinese Medicine

Chang Xu

Beijing University of Chinese Medicine

Jing Li

Beijing University of Chinese Medicine

Zhen-wei Liu

GuangXi University of Traditional Chinese Medicine

Feng Jiang

GuangXi University of Traditional Chinese Medicine

Xun Li

Beijing University of Chinese Medicine

Jian-Ping Liu ( $\nabla$ jianping_l@hotmail.com )

Beijing University of Chinese Medicine https://orcid.org/0000-0002-0320-061X

\section{Protocol}

Keywords: Chinese herbal medicine, HIV/AIDS-associated diarrhea, systematic review, meta-analysis, protocol

Posted Date: May 29th, 2020

DOI: https://doi.org/10.21203/rs.3.rs-31288/v1 
License: (c) (i) This work is licensed under a Creative Commons Attribution 4.0 International License. Read Full License 


\section{Abstract}

\section{Background:}

Diarrhea is a common symptom of acquired immune deficiency syndrome (AIDS) occurring either at the early stage of human immunodeficiency virus (HIV) infection or advanced stage, generally not fetal but seriously affect the quality of life.The treatment of HIV/AIDS-associated diarrhea based on traditional Chinese medicine (TCM) syndrome differentiation or combined with conventional therapy has showed better effect, and improve the immune status of patients to a certain extent, which may have a good application prospect. This is a protocol for a systematicreview on health care intervention. The objective is toassess the beneficial effect and safety of Chinese herbal medicine for people with HIV/AIDSassociated diarrhea.

\section{Methods:}

We will search and include randomized controlled trials comparing Chinese herbal medicine with placebo or other active interventions, regardless of language and publication status. The primary outcome will be diarrhea frequency (per day or per week) and fecal character. Databases to be searched include The Cochrane Central Register of Controlled Trials (CENTRAL) in the Cochrane Library; PubMed;China National Knowledge Infrastructure (CNKI); Wanfang Data; Chinese Biomedical Literature Database (SinoMed) and China Science and Technology Journal database (VIP). For each step, two reviewers will independently perform the selection of studies, data extractionand assess their methodological quality by the Cochrane risk of bias tool. We will analyze data, and performmeta-analyses if data available.

\section{Discussion:}

By providing current evidence of the beneficial effect and safety of Chinese herbal medicine for people with HIV/AIDS-associated diarrhea,we intend toidentify potentialtherapeutic modality that may be of benefits so to inform practice.

\section{Background:}

\section{Description of the condition:}

Acquired immune deficiency syndrome (AIDS) is a chronic infectious disease caused by human immunodeficiency virus (HIV). HIV mainly invades and damages CD4+T lymphocytes, resulting in impaired immune cells and defects, which may even cause severe opportunisticinfections and tumors ${ }^{[1]}$. According to the World Health Organization (WHO), as of 2018, about 39.7 million people were infected with HIV worldwide, most of whom live inlower-middle-income countries,and about 32 million people worldwide had died from AIDS-related causes ${ }^{[2]}$. Diarrhea isa common complication of AIDS and one of the primary causes of death among AIDS patients ${ }^{[3]}$. The clinical manifestations are mainly chronic diarrhea, and often accompanied by weight loss, malnutrition, wasting syndrome and even cachexia 
(weight loss, wasting of muscle, loss of appetite, and general debility ). The incidence of HIV/AIDSassociated diarrhea in North America and Europe is about $30 \%$ 80\%, with an incidence of up to $90 \%$ in developing countries ${ }^{[4]}$.

About $50 \% \sim 60 \%$ of HIV/AIDS-associated diarrhea is caused by opportunistic infection of viruses, bacteria, parasites and fungi ${ }^{[5]}$. The occurrence of diarrhea increases with a decrease of CD4+ T lymphocytes counts. It is therefore more common in areas with lower incomes wherehighly activeantiretroviral therapy (HARRT) is not available or affordable ${ }^{[6]}$. In addition, non-infectious causes such as malignant tumors, AIDS-related absorption disorders, AIDS-related individual idiopathic factors and side effects of HAART may exist during the whole process of AIDS progression ${ }^{[7-9]}$. In situations where HAART is commonly used, HIV/AIDS-associated diarrhea is more likely to exist as a side effect of HAART than a result of opportunistic infection ${ }^{[10][11]}$. The incidence of opportunistic infections causing diarrhea decreased from $53 \%$ to $13 \%$, whereas noninfectious causes of diarrhea increased from $32 \%$ to $70 \%{ }^{[12]}$. The predominant cause of noninfectious cases was medicationassociated diarrhea, which increased from $0 \%$ in 1995 to $45 \%$ in $1997^{[11]}$.

The most effective treatment for HIV infection has been proven to be HAART, which has significantly reduced the incidence and mortality of AIDS through the combination of three or more drugs ${ }^{[13]}$. However, HIV/AIDS-associated diarrhea is still very common. The diarrhea caused by HARRT drugs, especially the combination of drugs containing lopinavir/ritonavir, is worthy of attention. A study found that the proportion of HARRT drug-related diarrhea was as high as $65.60 \%$ among HIV/AIDS-associated diarrhea ${ }^{[14]}$. Due to the limitations of HAART, the long-term nature of drug administration, drug toxicity, drug resistance, and the inability to completely rebuild immune function ${ }^{[15]}$, the existing methods of modern medicine have poor therapeutic effect on HIV/AIDS-associated diarrhea, and there are some problems such as high toxicity, poor patient compliance, high recurrence rate and high price after drug withdrawal ${ }^{[16]}$. The therapeutic options of treating HIV/AIDS related complications such as diarrhea are still limited at present and alternative approaches are needed.

\section{Description of the intervention:}

Traditional Chinese Medicine (TCM) has developed in China for thousands of years. Chinese herbs are chosen because they come from natural medicinal plants, and the use of them as an essential medicine to treat diseases is often considered a natural, cheaper and more convenient alternative with fewer side effects.

The treatment of HIV/AIDS-associated diarrhea based on TCM syndrome differentiation or combined with conventional therapy has showed better effect, and can improve the immune status of patients to a certain extent ${ }^{[17][18]}$, which has a good application prospect, especially in areas where HAART is not available or economically feasible. 
There have been many clinical trials testing herbal medicines for HIV/AIDS-associated diarrhea during the past decades ${ }^{[19]-[26]}$. And in clinical practice in China, herbal medicine is commonly used for treating diarrhea ${ }^{[27]}$.

\section{How the intervention might work:}

Some clinical trials of traditional Chinese herbs in the treatment of HIV/AIDS-associated diarrhea have shown that theymay have the potential to relieve symptoms. TCM may reduce the local intestinal immune inflammatory injury, protect the intestinal mucosal barrier by increasing the levels of IL-17, IL-6, IL-23, IL-21 and other cytokines, so as to achieve the effect of treating diarrhea ${ }^{[28]}$.

There are several mechanisms of Chinese herbal medicine in treating diarrhea, the first one is to balance water and electrolytes in the body. For instance, Berberine(BBR) ${ }^{[29]}$, Atractylenolide $\mathrm{I}^{[30]}$, and Buzhong Yiqi Tang ${ }^{[31]}$ can up-regulate the expression of $\mathrm{Na}^{+} / \mathrm{H}^{+}$exchanger 3 and $\mathrm{Na}^{+} /$glucose cotransporter 1 to increase the absorption of water and $\mathrm{Na}^{+}$; Resveratrol dimer and resveratrol tetramers have the function of inhibiting the activity of cystic fibrosis transport regulator and calcium-activated chloride channel, so as to reduce the secretion of intestinal fluid by reducing the secretion of $\mathrm{Cl}^{-[32]}$; Rhubarb tannins can regulate aquaporins (AQP), inhibiting the expression of AQP2 and AQP3 to significantly decrease the faecal water content in colon ${ }^{[33]}$; Fuzi Lizhong Tang ${ }^{[34]}$ and Jiawei Renshen Wumei Tang ${ }^{[35]}$ can increase the expression of AQP4 to increase the intestinal absorption of water.

Secondly, it is by anti-inflammatory effects and regulation of immune responses that Chinese herbal medcine works. Some of the active ingredients of Chinese herbal medcine interfere nuclear factor kappaB, NOD (nucleotide binding oligomerization domain)-like receptor 3 (NLRP3), microRNA (ribonucleic acid)-155 (miR-155) and other signal pathways to regulate the secretion proinflammatory cytokines, and some can modulate the balance of different kinds of T-lymphocytes by means of promoting the apoptosis of $T$ helper cells, inhibiting mammalian target of rapamycin signal pathway, and raising the proportion of T regulatory cells ${ }^{[36]}$. For instance, BBR ${ }^{[37]}$,Polygonum hydropiper (Laliao) ${ }^{[38]}$, raw and branroasted Puerariae lobatae Radix (Gegen) ${ }^{[39]}$, Huangqin Tang ${ }^{[40]}$, Chrysanthemum morifolium Ramat (Juhua ${ }^{[41]}$ and Gegen Qinlian Tang ${ }^{[42]}$ can decrease level of proinflammatory cytokines such as interleukin-1 $\beta$ and tumor necrosis factor- $\alpha$; Shenlin Baizhu San ${ }^{[43]}$, Puerariae lobatae Radix (Gegen) ${ }^{[39]}$ and coked Atractylodes Rhizome(Cangzhu) ${ }^{[44]}$ can enhancethe level of anti-inflammatory cytokine interleukin-10to relieve inflammatory reaction.

Thirdly, it is by adjusting gastrointestinal motility and improving gastrointestinal function. For instance, Rhizoma Atractylodis (Baizhu) can affect the level of brain-gut peptide and the biosynthesis of neurotransmitters (or signal molecule), which are amino acid derivatives such as 5-hydroxytryptamine, gamma-aminobutyric acid and dopamine, by amino acid metabolism to affect the neuro-endocrine network homeostasis, so as to achieve the purpose of eliminating gastrointestinal dysfunction ${ }^{[45]}$; volatile 
oil extracted from Alpinia oxyphylla (Yizhi) can interfere in gastrointestinal hormone secretion, reducing the level of motilin and somatostain, to improve gastrointestinal function ${ }^{[46]}$.

Fourthly,it is by protecting intestinal epithelial barrier and promoting repair of intestine epithelial cells. For instance, SiJun Zi Tang can significantly enhance the expression of intestinal mucosal repair factor including proliferating cell nuclear antigen, epidermal growth factor receptor and transforming growth factor- $\beta 1^{[47]}$; patchouli alcohol can increase the expression of mucin- 1 and mucin-2 messenger RNA ${ }^{[48]}$; Gegen Qinlian Tang can up-regulate thefunction of intestinal barrier by enhancing the expression of tight junction proteins zonula occludens- 1 and occludin ${ }^{[42]}$; Decoction of compound Radix pulsatillae (Baitou Weng) can promote repair process of intestine epithelial cells by enhancing the activity of lactase $\mathrm{e}^{[49]}$.

Fifthly, it is by modulating the intestinal microbiota community.For instance, Atractylodes macrocephala (Baizhu) can significantly promote the proliferation rate of Lactobacillus acidophilus and inhibit the proliferation of Escherichia coli ${ }^{[50]}$; Chrysanthemum morifolium (Juhua) polysaccharides can elevate the abundances of Bifidobacterium, Butyricicoccus, Clostridium, Lachnospiraceae, Lactobacillus and Rikenellaceae, while opport- unistic pathogens (Enterococcus, Escherichia and prevotella) decrease ${ }^{[41]}$.

\section{Why it is important to do this review:}

Given the lack of effective treatment for HIV/AIDS-associated diarrhea, identifying existing therapeutic modality that may be of benefits is of high importance. There is a need for Chinese herbal medicine with an approved safety profile, supporting patients with HIV/AIDS-associated diarrhea through comprehensive treatment by relieving symptoms and side effects from antiretrovirals. But the evidence supporting the use of Chinese herbal medicine for treating HIV/AIDS-associated diarrhea is mainly theoretical and experiential. The quality and effects of the clinical trials should be systematically reviewed and appraised. Furthermore, a growing body of medical literature reports on hepatotoxicity and other adverse events in some herbal products ${ }^{[51][52]}$, as well as possible herb-drug interactions also warrant timely review ${ }^{[53]}$. The therapeutic effects, harms, and cost-effectiveness of Chinese herbs have not been well-established. Therefore, a systematic evaluation is of great importance.

\section{Objectives】}

To assess the beneficial effect and safety of Chinese herbal medicine for HIV/AIDS-associated diarrhea.

\section{Methods:}

\section{Criteria for considering studies for this review:}

\section{Types of studies:}


Randomized controlled trials(RCTs) comparing Chinese herbal medicine with placebo or other active interventions will be included, regardless of language and publication status.

\section{Types of participants:}

People with acute or chronic HIV/AIDS-associated diarrhea, regardless of race, age, gender or economic status will be included.

People with diarrhea caused by dysentery, cholera, poisoningand systemic disease will be excluded.

\section{Types of intervention:}

Experimental interventions are Chinese herbal medicine, including extracts from herb(s), single herbs or compoundformula, administered orally, taken either alone or in combination with other active treatment.

Control intervention can be no treatment, placebo, or other active treatment.

Co-intervention is allowed as long as it is applied in both groups.

\section{Types of outcome measures:}

\section{Primary outcomes:}

- Diarrhea frequency (per day or per week);

- Fecal character;

\section{Secondary outcomes:}

- Recovery durationof diarrhea;

- Other symptom such as fatigue and anorexia;

- Length of hospital stay;

- Body weight;

- Recurrence;

- Nutrition status;

- Quality of life (measured by validated tool or scale);

- Adverse effect: the incidence of alladverse events or adverse effects reported,includingwithdrawal from trial due to some adverse effects and modification of treatment plan. Based on the available data, we will classify the adverseevents as serious and non-serious. Any external medical event thatwas life-threatening, led to deathorsignificant or persistentdisability, or any medical occurrence which may have endangered patients or required intervention to prevent it belongs tothe serious adverse events ${ }^{[54]}$. Additionally, other adverse eventswill be considered non-serious. 
For the composite outcome index, only the results with the same components will be used for data analyses. If the composite outcome index is an ordered polytaxic variable, such as cure/significant effect/effective/ineffective, it will be dichotomized into effective/ineffective.

\section{Search methods for identification of studies:}

We will attempt to identify all relevant trials regardless of language or publication status (published, unpublished, in press, or in progress).

\section{Electronic searches:}

We will use the search terms and strategy described in Additional file 2 to search the following databases : The Cochrane Central Register of Controlled Trials (CENTRAL)in the Cochrane Library; PubMed;China National Knowledge Infrastructure (CNKI);Wanfang Data; Chinese Biomedical Literature Database (SinoMed); China Science and Technology Journal database (VIP).

\section{Searching other resources:}

We will examine all retrieved studies'reference lists and relevant reviews for further studies.

We will searchrelevant Chinese journals that aremostly likely to publish the trials aboutAIDS-related diarrhea by hand.

We will also contact researchers in the field to identify unpublished and ongoing studies.

\section{Data collection and analysis:}

For selection of studies and data extraction,two review authors (BLC and MZZ) will independently conduct each step, and examine agreement between them. We will resolve any disagreements through discussion with a third senior author (JPL).

\section{Selection of studies:}

Two review authors (ZWH and BLC) will check titles and abstracts retrieved by the searches of databases mentioned above independently. To make sure that we only include the same trial from multiple publications at once, we will examine each trial report. We will further evaluate the fulltext, if a record cannot be rejected with complete certainty for its title and abstract. We will contact the authors to clarify if the eligibility of a trial is unclear. We will resolve any disagreements through discussion, and involving a third author when necessary. We will also list the excluded studies as well as the reasons for their 
exclusion in a 'Characteristics of excluded studies' table. We will use a PRISMA diagram to display the study selection process.

\section{Data extraction and management:}

Retrieved citations from the various search engines will be imported into EndNote and checked for duplicates. Two review authors (BLC and MZZ) will use a pre-piloted data extraction form to extract data on general information (study ID, study author and title); methodological information (study design, the number of groups, sequence generation, allocation sequence concealment, blinding, selective outcome reporting, baseline comparability); participant characteristics (diagnostic criteria, inclusion criteria, exclusion criteria, acute/chronic, total number of intervention groups, number lost during follow-up, age, sex, country,setting and disease duration); intervention (the name of therapeutic drug, its form, dosage and regimen, other treatments given, drug combination, duration of treatment), and outcome measures.We will record relevant datausing a pre-defined data extraction form. We will resolve disagreements through discussion, and contact the corresponding trial author in case of unclear and missing data.

\section{Assessment of risk of bias in included studies:}

Two review authors (CX and HRZ) will assess methodological quality using the Cochrane 'Risk of bias' tool and report the results in a 'Risk of bias' table. We will use the criteria for judging risk of bias in the 'Risk of bias' assessment tool in the Cochrane Handbook for Systematic Reviews of Interventions, considering the following:

- Sequence generation.

- Allocation concealment.

- Blinding of participants, personnel and outcomes assessors.

- Incomplete outcome data.

- Selective outcome reporting. We hope that trials report adverse event data, survival and disease progression.

- Other possible sources of bias. We will assess vested interests depending on authors of thetrials and the source of funding. If a conflict of interests is obvious, the risk of bias will be judged If there is a clear declaration of a lack of conflict of interests, and there is no clear vested interest in the author or the funding,the risk of bias will be judged low.In the absence of a declaration, the risk of bias will be judged unclear.Additionally, we will assess baseline differences between groups. If the differences are not significant or exist, but the impact on the results has been investigated and found to be insignificant, the risk of bias will be judged low. If the differences are obvious and not addressed, the risk of bias will be judged high.In other cases, the risk of bias will be judged unclear. 
If information is unclear or not specified, we will also attempt to contact the trial authors. We will resolve any disagreements by discussion between the review authorsor, if necessary, by consulting another author.

\section{Measures of treatment effect:}

We will report dichotomous outcomes as risk ratios (RR) and 95\% confidence intervals (Cls). We will present continuous outcomes as mean differences (MD) and 95\% Cls if the outcomes have been measured in the same way across all included trials. We will use the standardized mean difference (SMDs) and $95 \% \mathrm{Cl}$ as the effect measureif included trials measured continuous outcomes in different ways. We will present time-to-event outcomes as hazard ratios and $95 \% \mathrm{Cls}$. We will also pay attention to the time points at which results were collected and reported.

\section{Unit of analysis issues:}

The unit of analysis will be individual patient in the case of individual trials. The unit of analysis will be the cluster in the case of a cluster randomized study design. If we identifycluster-randomized studies meeting the inclusion criteria, we will carry out appropriate analysis adjusting for the effect of cluster randomization before including effects estimates in a meta-analysis. If available, we will extract adjusted measures of effect from the trial reports. If only unadjusted data are available, we will adjust this data ourselves using the intracluster correlation coefficient (ICC). If the ICC is not reported, we will contact the study authors to obtain this, or borrow an ICC value from a similar study, or estimate the ICC. If the ICC is estimated, we will explore the robustness of analyses by conducting sensitivity analyses.

\section{Dealing with missing data:}

We will contact the trail authors to obtain the missing data. If it isnot successful, we will perform sensitivity analyses to investigate the impact of missing data on the primary outcomes. Data must lose credibility, at some degree of loss of follow-up ${ }^{[55]}$. However, wedetermined to include studies with more than $50 \%$ drop-out preliminarily due to insufficient evidence base up to now.If the trial reported the number of patients for whomthe primary outcomedata is missing, we will adopt a worst-case scenario approach toanalyze data.That is to say, patients with missing data in the control group will be considered treatment successes, while patients with missing data in the treatment group will be considered treatment failure ${ }^{[56]}$. We will indicate where studies have used imputation (and which methods) in this review.We will compare each protocol analysis of the available data with the one that contains missing data. We will conclude with more confidence in the case that the effect estimatesare the same and there are significant differences between groups. We will interpret the results more carefully and make more conservative conclusions about the effect of the treatment in the case that the effect estimates of the two analyses differ. 


\section{Assessment of heterogeneity:}

Heterogeneity will be assessed by visual examination of the forest plots, to determine the closeness of the point estimates to each other and the overlap of $\mathrm{Cls} \mathrm{Chi}^{2}$ test with a P value of 0.10 will be used to indicate statistical significance, and $\mathrm{I}^{2}$ statistic will be used to assess the heterogeneity with a value of $50 \%$ indicatingsubstantial heterogeneity ${ }^{[57]}$. If there is significant heterogeneity, we will use the random effects model, or we will choose the fixed effects model. The analyses will be carried out using Statistical analysis of the Cochrane software.

\section{Assessment of reporting biases:}

We will assess the potential impact of small studies such as publication bias by examining the funnel plots.If an adequate number of trials (more than 10 trials) are included in the meta-analysis, we will examine the symmetry of funnel plot. If the funnel plot is asymmetric, there may be publication bias.

\section{Data synthesis:}

We will use RevMan Web to analyze the data. We will use RR with $95 \% \mathrm{Cls}$ and a random-effects model to pool their results in the meta-analyses if there are sufficient clinically similar studies available. For dichotomous outcomes, we will calculate theRR for each study and then aggregate the data. For continuous outcomes, if all the trials measured results on the same scale, we will pool MDs between the treatment arms at the end of the follow-up, otherwise we will pool SMDs. Where a meta-analysis is inappropriate, we will summarize data in tables.

\section{Subgroup analysis and investigation of heterogeneity:}

We will perform the following subgroup analyses for people with AIDS-related diarrhea to investigate heterogeneity:

- Acute or chronic;

- Diarrhea cause by etiology;

- Type of interventions including but not limited to individual prescriptions of Chinese herbal medicine; a single herb or compound; dosage regimen;

- Sex, age and ethnicity.

\section{Sensitivity analysis:}


We will conduct sensitivity analyses to explore the impact of losses to follow-up on the effect estimates for the primary outcomes and to exclude studies considered to be high risk of bias. For dichotomous outcomes, we will vary the event rate within the missing patients from intervention and control groups within plausible limits. For continuous data, we will perform sensitivity analyses using methods described by Ebrahim 2013 and Ebrahim 2014[58] [59].

\section{Summary of findings and assessment of the certainty of the evidence:}

We will use the Grading of Recommendations Assessment, Development and Evaluation (GRADE) approach to evaluate the quality of evidence and present it in 'Summary of Findings' tables. We willassess the certainty of a range of evidence which associated with the studies providing data for metaanalyses of prespecified outcomes by using the five GRADE considerations (study limitations, effectconsistency, imprecision, indirectness and publication bias) ${ }^{[56]}$. We will use methods and recommendations described in Chapter 14 of the Cochrane Handbook for Systematic Reviews of Interventions ${ }^{[60]}$, and use GRADEpro software. We will use footnotes to justify all decisions that downgrade the certainty of evidenceandmake comments where necessaryto help readers understand the review.

\section{Discussion:}

The strengths of this project lie in its value for guiding clinical practice and find out innovative therapeutics for HIV/AIDS-associated diarrhea.There is a need for Chinese herbal medicine with an approved safety profile, supporting patients with HIV/AIDS-associated diarrhea through comprehensive treatment by relieving symptoms and side effects from antiretrovirals.

This protocol has some limitations too. Some studies on the efficacy of Chinese herbal medicine in the treatment of HIV/AIDS-associated diarrhea has been reported, but is generally of poor quality. And there are large variationsamong tested herbal remedies that it is difficult to conduct meta-analysis.Additionally, the search strategy is comprehensive and does not limit the language, but there is still a lack of sufficient English Literature, which may be because the acceptance of TCM treatment in foreign countries is not as good as in China, so as to may affect the extrapolation of the research results. Therefore, we look forwardto more high-qualityand large-sample RCTs in depth to support our review results and toinform the international audience about Chinese herbal medicine in the treatment of HIV/AIDS-associated diarrhea.

\section{Abbreviations:}

AIDS:Acquired immune deficiency syndrome 
HIV:Human immunodeficiency virus

WHO:World Health Organization

HAART:Highly activeanti-retroviral therapy

TCM:Traditional Chinese medicine

BBR: Berberine

AQP:Aquaporins

NOD:Nucleotide binding oligomerization domain

NLRP3: NOD-like receptor 3

RNA:Ribonucleic acid

MiR-155:MicroRNA-155

PRISMA:Preferred Reporting Items for Systematic Reviews and Meta-Analyses

RCTs:Randomized controlled trials

RR: Risk ratios

Cls:Confidence intervals

MD: Mean differences

SMDs: Standardized mean difference

ICC: Intracluster correlation coefficient

\section{Declaration:}

\section{Ethics approval and consent to participate:}

Not applicable.

\section{Consent for publication:}

We would like to submit our full review to an international peer review journal.

\section{Availability of data and materials:}


The datasets used and/or analysed in the reviewwould be available from the corresponding author on reasonable request.

\section{Competing interests:}

The authors declare that they have no competing interests.

\section{Funding:}

This work was supported by theNational Natural Science Foundation of China (Grant No.: 81673828).

\section{Authors' contributions:}

BLC and JPL conceived and coordinated this project. All authors contributed to the development of the protocol. BLC developed the search strategies. All authors will screen the retrieved studies. BLC drafted the protocol, and all authors critically reviewed and approved the final version. BLC is the guarantor of this protocol.

\section{Acknowledgements:}

Not applicable.

\section{References}

1. [1] WHO. HIV/AIDS[Online]. 2019 [updated October 2019. [Available from: https://www.who.int/health-topics/hiv-aids/\#tab=tab_1

2. [2] WHO. 10 FACTS ON HIV/AIDS [Online]. 2019 [updated July 2019. [Available from: https://www.who.int/news-room/facts-in-pictures/detail/hiv-aids.

3. [3] Yan L, Guo HJ. The research progression of AIDS diarrhea treated with TCM. Liaoning Journal of Traditional Chinese Medicine,2011,38(12):2490-3.

4. [4] Lew EA, Poles MA, Dieterich DT. Diarrheal diseases associated with HIV infection. Gastroenterology clinics of North America. 1997;26(2):259-90.

5. [5] WHO. Interim WHO clinical staging of HIV/AIDS and HIV/AIDS case definitions for surveillance 2005 [updated June 2005. [Available from: https://www.who.int/hiv/pub/guidelines/ casedefinitions/en/.

6. [6] Megan E OB. Patterns and correlates of discontinuation of the initial HAART regimen in an urban outpatient cohort[J]. Journal of acquired immune deficiency syndromes.2003;34(4):407-14. 
7. [7] AIDS WHOGPo. Guidelines for the clinical management of HIV infection in adults. Geneva: World Health Organization; 1991.

8. [8] Macarthur RD. Management of noninfectious diarrhea associated with HIV and highly active antiretroviral therapy. The American journal of managed care. 2013;12 Suppl (19):s238-45.

9. [10]Pape JW, Verdier RI, Boncy M, Boncy J, Johnson WD, Jr. Cyclospora infection in adults infected with HIV. Clinical manifestations, treatment, and prophylaxis. Annals of internal medicine.1994;121(9):654-7.

10. [11]Logan C, Beadsworth MB, Beeching NJ. HIV and diarrhoea: what is new? Current opinion in infectious diseases. 2016;29(5):486-94.

11. [12]Call SA, Heudebert G, Saag M, Wilcox CM. The changing etiology of chronic diarrhea in HIVinfected patients with CD 4 cell counts less than 200 cells $/ \mathrm{mm} 3$. Am J Gastroenterol.2000;95:3142-6.

12. [14] Zhang XW, Zhang AP, Li Z, et al.The analysis of the clinical features of 67 cases of HIV/AIDS related diarrhea.The Chinese Journal of Dermatovenereology,2016,36(7):712-3】

13. [19] Li Z, Yuan J, Zhang XW, et al. 19 cases of AIDS-related diarrhea with spleen-kidney yang deficiency treated by Jianpi Zhixie decoction. Traditional Chinese Medicinal Research,2016,29(8):113.

14. [20] Dong SQ.Clinical research of Xieli Kang capsule treatment of chronic diarrhea. Acta Chinese Medicine,2014,29(11):1549-51.

15. [21] Xu Z, Yang XP, Ni L, et al.Clinical study on Xielikang capsule in treatment of AIDS-related chronic diarrhea. Global Traditional Chinese Medicine,2011,4(3):197-200.

16. [24] Dang ZQ. 56 casesof Modified Chishizhi Yuyuliang decoction to treat AIDS refractory diarrhea.Chinese Journal of Basic Medicine in Traditional Chinese Medicine,2013,19(7):844凶5.

\section{Supplementary Files}

This is a list of supplementary files associated with this preprint. Click to download.

- Additionalfile2.docx

- Additionalfile1.docx 